\title{
Conceptual Model for WWBAN (Wearable Wireless Body Area Network)
}

\author{
Jawad Hussain Awan \\ Institute of Information and \\ Communication Technology \\ University of Sindh, \\ Jamshoro, Pakistan
}

\author{
Shahzad Ahmed Memon \\ Institute of Information and \\ Communication Technology \\ University of Sindh, \\ Jamshoro, Pakistan
}

\author{
Nisar Ahmed Memon \\ Institute of Information and \\ Communication Technology \\ University of Sindh, \\ Jamshoro, Pakistan
}

\author{
Raza Shah \\ Institute of Information and \\ Communication Technology \\ University of Sindh, \\ Jamshoro, Pakistan
}

\author{
Zulifqar Bhutto \\ Institute of Information and \\ Communication Technology \\ University of Sindh, \\ Jamshoro, Pakistan
}

\author{
Rahat Ali Khan \\ Institute of Information and \\ Communication Technology \\ University of Sindh, \\ Jamshoro, Pakistan
}

\begin{abstract}
Modern world advances in sensors miniaturization and wireless networking which enables exploiting wireless sensor networking to monitor and control the environment. Human health monitoring is promising applications of sensor networks in a healthcare environment. Sensor system was worn by the human that creates wireless body area network to monitor and provide synchronized response to the patients for medical contextual information received by sensors. Though, challenging tasks are encountered by researchers to address habitually conflicting necessities for size, time to operate, correctness of data, reliability and time to store that data and provide responses accordingly.

This paper encompasses the structural design of hardware as well as software in a wireless sensor network system for monitoring health issues. The paper outlines few healthcare services, innovations latest trends those monitor patients in the health care systems and propose some of the other future trends where they might be helpful for future research to be used in handheld.
\end{abstract}

Keywords-Healthcare Environment; Healthcare service; wireless body area networks and wireless sensors

\section{INTRODUCTION}

In this modern world, population ratio is increasing day by day, which would reach 600 million by 2020 [1] and health diseases are also increases. In this way, Health care costs have been reduced by providing more skilled physicians, reduced hospital stays as well as skill level and regularity home-care professional's visit and promoting health education [5]. Furthermore, it is also discussed that healthcare system provides synchronized monitoring, premature diagnosis, and potential risky diseases treatments.

Besides, wireless communication medium delivers the medical diagnosis and patient consultations. In this regard, healthcare system is becoming cheaper and smarter which manages and cares for patients who are suffering from different diseases, such as heart syndrome [2] and long standing monitoring is proceeding rather than periodic assessments for constant persistent diseases.
In 2005, Jianchu Yao stated that an uninterrupted health monitoring system is easy to use and conductive too [3]. So, a wearable and plug-and-play system using Bluetooth is proposed. "A mobile patient monitoring system is proposed and integrates existing personal digital assistant (PDA) and Wireless Local Area Network (WLAN) technology" as stated in 2003 [4] and illustrates about wireless PDA model, which is enhanced suitable for patient transportation and mobility. In [5] health facility is provided by risk patient monitoring system in daily living environment.

A wearable healthcare system requires several technologies for implementation, where user's physiological signals are measured by a physiological signal measurement technology and sensor network provides a WBAN (Wireless Body Area Network) as well as healthcare systems for critical patients.

\section{BACKGROUND}

Wearable and sensor technology [6] [7] is an emerging technology that is implemented in body area networks as well as in pervasive systems to provide efficient and reliable services. Currently, it is noticed that WBAN [8] security as well as its protocol issues is under research. Few research directions [9] have implanted Body Area Network (BAN) systems for the monitoring of patients. Pervasive health [[10], [11] is playing an important role in patient monitoring via ubiquitous health. Ubiquitous healthcare programs for stroke patients have been started to monitor patients as well as activity detection [12] implemented a multi-accelerometer system, which is collaborative project between Stephen Intille and MIT (Massachusetts Institute of Technology).

Some challenges and their overview about WBAN and WSN are discussed in the table I which is taken from Hafez Fouad 2014 [13].

\section{REQUIREMENTS FOR WBAN}

This section is categorized into two sub sections. One of them is describing requirements for WBAN system 
applications and second section is comprised of technology standards [14]. In this sub section, some requirements for WBAN system applications have been discussed. Most common and world known application is ECG (Electro Cardio Gram) which has $144 \mathrm{kbps}$ of data rate, $100 \mathrm{~Hz}$ to $1 \mathrm{kHz}$ bandwidth, less than $250 \mathrm{~ms}$ latency, 12 bits accuracy. Second application is EMG (Electro Moy Gram) which has $300 \mathrm{kbps}$ of data rate, $0 \mathrm{~Hz}$ to $900 \mathrm{~Hz}$ bandwidth, less than $250 \mathrm{~ms}$ latency, 16 bits accuracy. Third application is EEG (Electro Encephala Gram) which has $43.2 \mathrm{kbps}$ of data rate, $0-150 \mathrm{~Hz}$ bandwidth, less than $250 \mathrm{~ms}$ latency, 12 bits accuracy. Fourth application is Blood saturation which has 16bps of data rate, 0-1 Hz bandwidth, 8 bits accuracy. Fifth, activity sensor application has $32 \mathrm{bps}$ of data rate, $0-480 \mathrm{~Hz}$ bandwidth, 12 bits accuracy. Sixth, Real time applications have $10 \mathrm{Mbps}$ of data rate, less than $105 \mathrm{~ms}$ latency. Seventh, Capsule endoscope applications have $1 \mathrm{Mbps}$ data rate. Eighth, Artificial retina applications have 50-700 kbps data rate. Ninth and final, Cochlear implant applications have $100 \mathrm{kbps}$ data rate. All the above mentioned applications have 10-10 reliability except Real time and activity sensor applications that have 10-3 reliability.

TABLE I. OVERVIEW OF WSN AND WBAN [13]

\begin{tabular}{|c|c|c|}
\hline Challenges & WSN & WBAN \\
\hline Scale & $\begin{array}{l}\text { Monitored in meters as } \\
\text { well as kilometers. }\end{array}$ & $\begin{array}{l}\text { Human body } \\
\text { (centimeters/meters) }\end{array}$ \\
\hline $\begin{array}{l}\text { Number of } \\
\text { nodes }\end{array}$ & Redundant nodes. & Fewer nodes. \\
\hline Accuracy & By node redundancy & $\begin{array}{l}\text { By node precision and } \\
\text { robustness }\end{array}$ \\
\hline Tasks & $\begin{array}{l}\text { Dedicated task is } \\
\text { performed }\end{array}$ & $\begin{array}{l}\text { Multiple tasks are performed } \\
\text { by nodes }\end{array}$ \\
\hline Size & Smaller size & Smallest size \\
\hline Network & Fixed or static & Variable \\
\hline Data rates & Homogeneous & Heterogeneous \\
\hline $\begin{array}{l}\text { Replacement } \\
\text { of nodes }\end{array}$ & $\begin{array}{l}\text { Easy to replace the } \\
\text { nodes. }\end{array}$ & $\begin{array}{l}\text { Difficult to replace implanted } \\
\text { nodes. }\end{array}$ \\
\hline Scale & $\begin{array}{l}\text { Monitored in meters as } \\
\text { well as kilometers. }\end{array}$ & $\begin{array}{l}\text { Human body } \\
\text { (centimeters/meters) }\end{array}$ \\
\hline Lifetime & Several years/months & Several years/months \\
\hline Power & $\begin{array}{l}\text { Easy to supply large } \\
\text { energy. }\end{array}$ & Supply smaller energy. \\
\hline $\begin{array}{l}\text { Scavenging } \\
\text { source }\end{array}$ & Solar and wind power & $\begin{array}{l}\text { Motion (vibration) and thermal } \\
\text { (body heat). }\end{array}$ \\
\hline Security & Lower level & More significant level \\
\hline Impact & $\begin{array}{l}\text { compensated by } \\
\text { redundant nodes }\end{array}$ & $\begin{array}{l}\text { Guarantee the delivery of QoS } \\
\text { (Quality of Service) and real } \\
\text { time data. }\end{array}$ \\
\hline Technology & $\begin{array}{l}\text { Bluetooth, Zigbee, } \\
\text { GPRS(General Packet } \\
\text { Radio Service), } \\
\text { WLAN(Wireless } \\
\text { Local Area Network) }\end{array}$ & $\begin{array}{l}\text { Requires Low power } \\
\text { technology }\end{array}$ \\
\hline
\end{tabular}

In this sub section, few technology standards have been discussed and compared according to their requirements, networking topology, security and device/application complexity.

Wi-Fi (Wireless Fidelity) standard has 100 meter distance converge, 11 to $54 \mathrm{Mbps}$ of data rate, $2.4 \mathrm{GHz}$ frequency, requires $5 \mathrm{GHz}$ to $20 \mathrm{MHz}$ bandwidth, requires high power, Point-Hub networking topology, AES block cipher and 32 bit $\mathrm{CRC}$ for security with high complexity. Bluetooth standard has 10 meter distance converge, $1 \mathrm{Mbps}$ of data rate, $2.4 \mathrm{GHz}$ frequency, requires $1 \mathrm{MHz}$ bandwidth, consumes medium power, Ad hoc networking, 64 and 128 bit encryption and 16 bit CRC for security having high complexity. UWB (Ultra Wide Band) standard has 10 meter distance converge, 100 to $500 \mathrm{Mbps}$ of data rate, 3.1 to $10.6 \mathrm{GHz}$ frequency, requires less than $500 \mathrm{MHz}$ bandwidth, consumes low power, Point-toPoint networking, ES block cipher and 16 bit CRC for security with Medium complexity. ZigBee standard has 70 to 100 meter distance converge, $250 \mathrm{Kbps}$ of data rate, $2.4 \mathrm{GHz}$ frequency, requires $2 \mathrm{MHz}$ bandwidth, very low power consumer, (Ad hoc, Peer to Peer, Mesh or star) networking, 128 AES with application layer security for security along with low complexity. WiMax (Worldwide Interoperability for Microwave Access) standard has 50 meter distance converge, $75 \mathrm{Mbps}$ of data rate, 2 to $11 \mathrm{GHz}$ frequency, requires 10 $\mathrm{MHz}$ bandwidth, requires low power, Infrastructure networking, and AES triple data encryption standard for security along with low complexity. WiBro (Wireless Broadband) standard has less than 2 miles distance converge, 1 to $75 \mathrm{Mbps}$ of data rate, 2.3 to $2.4 \mathrm{GHz}$ frequency, requires $8.75 \mathrm{MHz}$ bandwidth, requires low power, (Infrastructure and mesh) networking, AES with extensible authentication protocol for security with low complexity. Wireless USB (Universal Serial Bus) standard has 10 meter distance converge, $480 \mathrm{Mbps}$ of data rate, 3.1 to $10.6 \mathrm{GHz}$ frequency, requires $528 \mathrm{MHz}$ bandwidth, consumes low power, Point-toPoint networking, AES 128 for security with low complexity. Wireless IR (Infra-Red) standard has less than 10 meter distance converge, with LOS(Line Of Sight) $4 \mathrm{Mbps}$ of data rate, $16 \mathrm{KHz}$ frequency, requires $2.54 \mathrm{MHz}$ bandwidth, requires low power, Point-to-Point networking, very secure for security having low complexity.

\section{CONCEPTUAL ARCHITECTURE OF PROPOSED SYSTEM}

In this modern technological world, some artificial environments are designed to facilitate the human who implant this type of environment for security, health and communication. Because of these, the healthcare system is proposed which is comprised of following:

- IEEE 802.15.6 Standard.

- Sensor system \& Computer/nodes.

- Communication medium.

- Scanning algorithm.

\section{A. IEEE 802.15.6 Standard}

IEEE 802.15.6 standard [15] has been established by IEEE 802 Task Group 6 (TG6) for the standardization of WBAN. IEEE 802.15.6 is a communication standard optimized for low-power in-body/on-body nodes to serve a variety of medical and non-medical applications [16]The operating frequencies are defined for implanted devices. Such as $402-$ $405 \mathrm{MHz}$ and on-body devices which have $2.5 \mathrm{GHz}$ ISM and $3.1-10.5 \mathrm{GHz}$ UWB frequency bands.

The healthcare [17] is helpful for the scenarios Such as: Implant to Implant, Implant to Body surface, Implant to external, Body surface to Body surface and Body surface to External. 


\section{B. Sensor system \& Computer/nodes}

In this proposed system, sensor and computers are utilized for communication using IEEE 802.15.6 and sensor collects contextual information which processes the physiological signals to generate result-oriented signals that are transmitted to computers. Result-oriented signals information is manipulated by computers into abstracted information. As contextual information is collected that has to convert into higher level context using scanning methods at preliminary stage.

Proposed healthcare system provides services to patients simultaneously and system has to connect with service provider via communication channel shown in fig: 1 .

Fuel band sensor system and mCube accelerometer (clothing sensor) system have been proposed. mCube accelerometer is more accurate, more power-efficient, and cheaper chip that is comprised of two tiny chips (mechanical and microchip). A mechanical chip that detects movement and second microchip that makes sense of the signal from the first chip [18].

Fuel band is a sensor device that tracks and measures the activities those taken place by human interaction. Fuel band looks like a bracelet having energy efficiency, low energy radio, long battery life (for week), fitness tracking capability, sensing and monitoring ability[19].

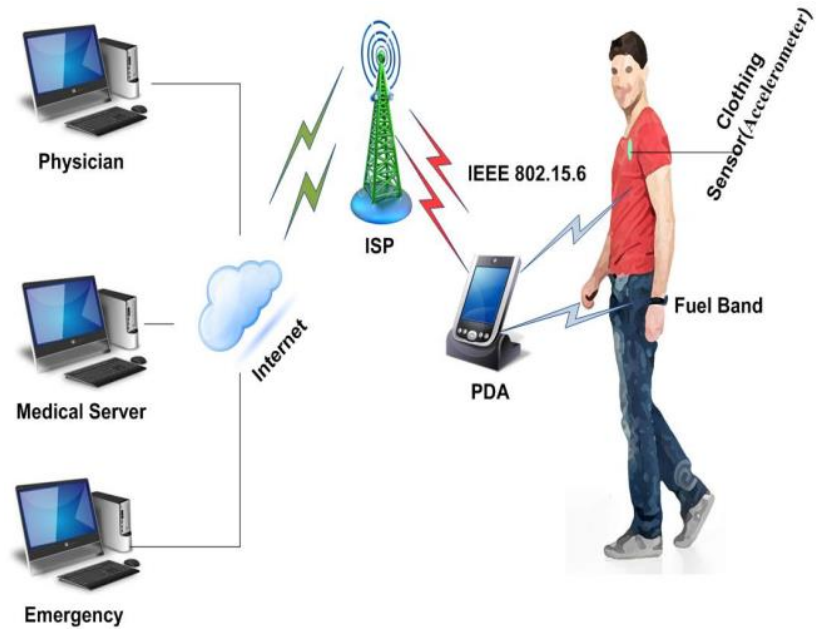

Fig. 1. Conceptual architecture of the proposed network

\section{The Computer and Communication Modules}

In this section, wearable PDA (Personal Digital Assistant) [14] is used as a wearable computer which has compatibilities. Such as: $480 \mathrm{MHz}$ XScale processor, a $128 \mathrm{MB}$ RAM, IEEE 802.15 .6 and a serial port.

The IEEE 802.15.6 plays an important role as communication medium among sensors, service providers and other context sources via WLAN. The proposed system, which manipulates result-oriented signals information into abstracted information. For interoperability, information is coming from sensors and service providing entities are transferred to the computer. In this way, Patients are monitored through collected information and treated accordingly.

\section{Scanning algorithm}

The proposed algorithm [20] is IEEE 802.15.4, which is used to establish a connection between PDA having compatibility of PSD (Physiological Signal Device) and sensor system for WBAN application in which sensor system channel is working on the basis of channel priority. The PSD contains past connection information which and when sensor system was connected and what activity was performed. In this way, PSD also knows about channel priority of sensor system. Thus, it doesn't require any scanning for sensor systems during connectivity.

The scanning time in the proposed algorithm for finding the sensor system where $\mathrm{Pi}$ is probability at $\mathrm{i}$ channel shown in following equation is taken from Joonyoung Jung 2008 [1]

$$
\sum_{i=1}^{i=l a s t}\{\operatorname{Pi} \times[(i-1 / 2) \times(\operatorname{Sx}(2 n+1))](\text { Symbols })\}
$$

Where S: aBaseSuperframeDuration,

$\mathrm{n}$ : Scan Duration $(0-14)$,

Pi: $i^{\text {-th }}$ Channel Probability.

\section{Health CARE SERVICES}

In this proposed model, two services either remote or local services have been proposed for healthcare system.

\section{A. Local Service}

Local services are provided by wearable devices, which are supervised by users with Personal Area Network (PAN) or Home Area Network (HAN) networks. This wearable device or node executes a code which is available for downloading using Wireless Local Area Network (WLAN) for local service. Wearable computer carries manipulated context, where a service is provided accordingly shown in fig: 2 .

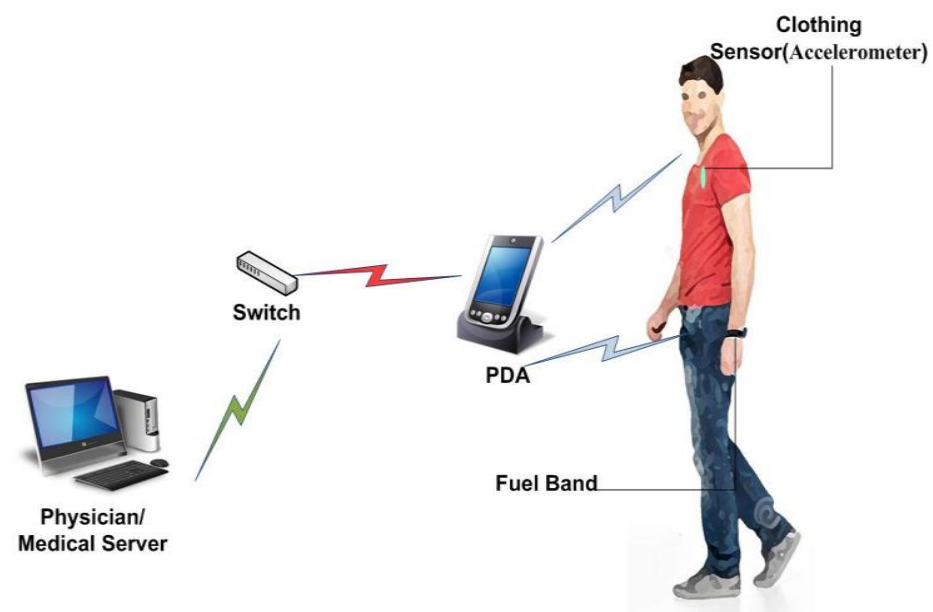

Fig. 2. Local Service for healthcare system

\section{B. Remote Service}

Remote healthcare service is supervised and operated by service provider and lies in remote servers. These remote servers contain service entities and their contextual information which is transferred via WAN, which plays vital role for the healthcare remote service shown in fig: 3 . 


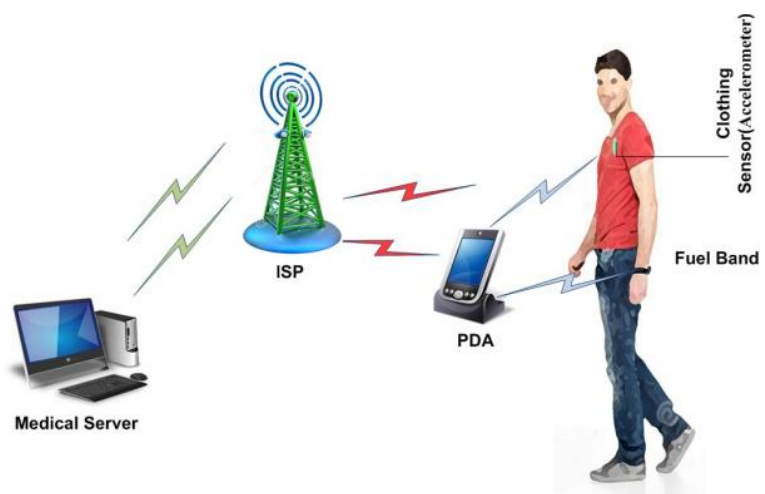

Fig. 3. Remote Service for healthcare system

\section{INNOVATION AND LATEST TRENDS}

Telemedicine is the remote medical service by which clinical services are provided via internet, telephone and videoconferencing [21]. Telemedicine service is fruitful for remote region communities where patients have to cover huge distance for medical treatment to doctors. Telemedicine has also eliminated the infection ratio of medical staff from transmission like MRSA (Methicillin Resistant Staphylococcus Aureus) with the help of telemedicine. White chrome syndrome patient gets relax through remote treatment.

Telemedicine is categorized into three services.

- $\quad$ Store and forward.

- Remote monitoring.

- Interactive service.

\section{A. Store and forward service}

Store and forward service is an asynchronous telemedicine service in which patient medical information. Such as Physiological signals and history reports which are transmitted to doctor for assessment offline. Such as: Tele-dermatology and Tele-pathology.

\section{B. Remote monitoring service}

Remote monitoring service is a synchronous telemedicine service in which patient is monitored via webcam. Remote monitoring is cost-effective as well as most favorable for chronic disease patients. Such as: Heart beat and Asthma.

\section{Interactive service}

Interactive service is real time healthcare service in which patients and clinical service provider communicated via voice conversation. History review, psychiatric and Ophthalmology assessments are conducted to assess the patient. Such as MDPhone (Med Phone) is an interactive system.

Tele-nursing is also part of telehealth application which provides distance consultancy of nurse via telecommunication channels [22]. The growth ratio of tele-nursing is increased because of healthcare to rural populated regions. Tele-nursing saves travel time and keeps patients at their homes for consultancy. Google Glass Breastfeeding is the first handsfree application, by which mothers are instructed to nurse their babies via Google hangout.
Tele-surgery is also remote service where patient's surgical performance is assessed by a robotic tele-operator surgeon controlled system [23]. This remote service allows surgeons to do surgical activities remotely at far distance. Tele-surgery requires robotics, communication technology as well as management information systems to provide surgeon activities remotely.

Tele-radiology is an interpretation between doctor and patient by transmitting of radiological images such as X-rays and MRI (Magnetic Resonance Imaging) via telecommunication channels [24]. A radiological image sending station, a transmission network and a receiving image station are key components to establish a tele-radiology service. In this health care service, a radiological signal is scanned at sending station and transmitted through transmission network and received at receiving image station where radiological images are reviewed for assessment.

E-heath is an emerging health technology which is supported by electronic processes and communication [25]. Ehealth service is mostly deployed in European countries. Such as: United Kingdom where E-health is termed as an umbrella in healthcare services.

\section{FUTURE WORK}

WBAN is an emerging technology that helps in the monitoring of peoples' healthcare and provides them reliable faster services in emergency. So, researchers are sharing their ideas about new directions for WBAN. Few of them are defined in this section for future work. In hardware design, low power sensors are under development by harvesting energy from body movements or using technique to generate its power. Interconnection feature of WBAN should be developed intelligently. So that WBAN node can adopt itself in environment is also new direction for researchers. A dedicated wireless transmission standard is being tried for implementing in WBAN to eliminate interference issues. Fully autonomous, long life and multimedia systems are under development which can support advance medical healthcare diagnostic systems.

\section{CONCLUSION}

In this paper, a conceptual model for WWBAN healthcare system is proposed. A WWBAN which uses IEEE 802.15.6 standard, sensor technology and wearable devices, those have been employed to monitor the patient health and their activity accordingly and react whenever any conflict or emergency occurs. Sensor nodes and wearable devices Such as: fuel band or clothing sensor are capable of emitting physiological signals which are transmitted to service provider server via networks i-e WLAN, WAN, PAN and HAN. Proposed health care system is useful for medical healthcare environment and comprises of sensor systems which transmit bio physiological signals to computer and communication modules that converts them into abstracted information and then health care services are communicated either locally or remotely. Few new trends have also been discussed to motivate the researchers and technical personals to design new approaches, which can be helpful for patients in emergency conditions. 


\section{REFERENCES}

[1] J. Jung, K. Ha, J. Lee, Y. Kim, and D. Kim, "Wireless Body Area Network in a Ubiquitous Healthcare System for Physiological Signal Monitoring and Health Consulting."

[2] Lubrin, E. Lawrence, and K. F. Navarro, "Wireless Remote Healthcare Monitoring with Motes," in International Conference on Mobile Business (ICMB'05), pp. 235-241.

[3] J. Yao, R. Schmitz, and S. Warren, "A Wearable Point-of-Care System for Home Use That Incorporates Plug-and-Play and Wireless Standards," IEEE Trans. Inf. Technol. Biomed., vol. 9, no. 3, pp. 363371, Sep. 2005.

[4] Y.-H. Lin, I.-C. Jan, P. C.-I. Ko, Y.-Y. Chen, J.-M. Wong, and G.-J. Jan, "A Wireless PDA-Based Physiological Monitoring System for Patient Transport," IEEE Trans. Inf. Technol. Biomed., vol. 8, no. 4, pp. 439447, Dec. 2004.

[5] N. Golmie, D. Cypher, and O. Rebala, "Performance analysis of low rate wireless technologies for medical applications," Comput. Commun., vol. 28, no. 10, pp. 1266-1275, 2005.

[6] Arya and N. Bilandi, "A Review: Wireless Body Area Networks for Health Care," Int. J. Innov. Res. Comput. Commun. Eng. (An ISO Certif. Organ., vol. 3297, no. 4, 2007.

[7] Arya, S. Pathania, and C. Kaushal, "Cloud-Based Wireless Body Area Network for Healthcare Monitoring System,” vol. 2, no. 8, pp. 23939907.

[8] S. N. Ramli and R. Ahmad, "Surveying the Wireless Body Area Network in the realm of wireless communication," in 2011 7th International Conference on Information Assurance and Security (IAS), 2011, pp. 58-61.

[9] D. Kurjekar and N. M. Palekar, "Ubiquitous Healthcare Monitor System Using Wearable Wireless Sensor Network."

[10] J. K.-Y. Ng and S. M. of IEEE, "Ubiquitous Healthcare: Healthcare Systems and Applications enabled by Mobile and Wireless Technologies," JoC, vol. 3, no. 2, pp. 31-36.

[11] K. Dey and D. Estrin, "Perspectives on Pervasive Health from Some of the Field's Leading Researchers," IEEE Pervasive Comput., vol. 10, no. 2, pp. 4-7, Apr. 2011.

[12] W. Kaiser and M. Sarrafzadeh, "Introduction to special issue on wireless health,” ACM Trans. Embed. Comput. Syst., vol. 10, no. 1, pp. 1-1, Aug. 2010.

[13] H. Fouad, "Patient-Oriented Web Telemedicine System for Health Monitoring,” J. Commun. Comput., vol. 11, pp. 168-178, 2014.
[14] Chakraborty, B. Gupta, and S. K. Ghosh, "A Review on TelemedicineBased WBAN Framework for Patient Monitoring," Telemed. e-Health, vol. 19, no. 8, pp. 619-626, Aug. 2013.

[15] S. Saleem, S. Ullah, and K. S. Kwak, "A Study of IEEE 802.15.4 Security Framework for Wireless Body Area Networks," Sensors, vol. 11, no. 12, pp. 1383-1395, Jan. 2011.

[16] Kenichi Takizawa, Takahiro Aoyagi, Jun-ichi Takada, Norihiko Katayama, Kamya Yekeh, Yazdandoost Takehiko, and Kobayashi Ryuji Kohno, "Channel models for wireless body area networks," in 2008 30th Annual International Conference of the IEEE Engineering in Medicine and Biology Society, 2008, pp. 1549-1552.

[17] H. Viittala, M. Hamalainen, and J. Iinatti, "Different experimental WBAN channel models and IEEE802.15.6 models: Comparison and effects," in 2009 2nd International Symposium on Applied Sciences in Biomedical and Communication Technologies, 2009, pp. 1-5.

[18] Cuthbertson, "World's Smallest Motion Sensor Could Transform Everyday Clothes into Electronics," 2014. [Online]. Available: http://www.ibtimes.co.uk/worlds-smallest-motion-sensor-couldtransform-everyday-clothes-into-electronics-1461770. [Accessed: 05Dec-2015].

[19] Mat Smith, "Nike FuelBand SE review: more social features, much longer battery life." [Online]. Available: http://www.engadget.com/2013/11/27/nike-fuelband-se-review/. [Accessed: 10-Mar-2015].

[20] J. Jung, K. Ha, and J. Lee, "Wireless Body Area Network in a Ubiquitous Healthcare System for Physiological Signal Monitoring and Health Consulting," pp. 47-54.

[21] Pinciroli, M. Corso, A. Fuggetta, M. Masseroli, S. Bonacina, and S. Marceglia, "Telemedicine and E-Health," IEEE Pulse, vol. 2, no. 3, pp. 62-70, May 2011.

[22] L. Schlachta-Fairchild, V. Elfrink, and A. Deickman, Patient Safety, Telenursing, and Telehealth. 2008.

[23] R. Jiménez Moreno, F. A. Espinosa Valcárcel, and D. Amaya Hurtado, "TELEOPERATED SYSTEMS: A PERSPECTIVE ON TELESURGERY APPLICATIONS,” Rev. Ing. Biom\{é\} dica, vol. 7, no. 14, pp. 30-41, 2013.

[24] S. Spijker, "Teleradiology quality assurance--lessons learnt," Pediatr. Radiol., vol. 44, no. 6, p. 704, 2014.

[25] Y.-P. Chen, C.-K. Liu, C.-H. Chen, T.-F. Huang, S.-T. Tu, and M.-C. Hsieh, "The Investigation on Effect of Tele-Care Combined Dietary Reminds in Overweight Cases," 2014, pp. 2253-2258. 